\title{
The analysis of Abell 1835 using a deprojection technique
}

\author{
S. M. Jia ${ }^{1,2}$, Y. Chen ${ }^{1}$, F. J. Lu ${ }^{1}$, L. Chen ${ }^{1,2}$, and F. Xiang ${ }^{1}$ \\ 1 Particle Astrophysics Center, Institute of High Energy Physics, Chinese Academy of Sciences, Beijing 100039, PR China \\ e-mail: jiasm@mail.ihep.ac.cn \\ 2 Department of Astronomy, Beijing Normal University, Beijing 100875, PR China
}

Received 26 June 2003 / Accepted 1 April 2004

\begin{abstract}
We present the results from a detailed deprojection analysis of Abell 1835 as observed by XMM-Newton. If we fit the spectra with an isothermal plasma model, the deprojected temperature profile is flat in the outer region around $7.6 \mathrm{keV}$ and decreases to $\sim 5.6 \mathrm{keV}$ in the center, which may be connected with the gas cooling. In the central part, a two-component thermal plasma model can fit the spectrum significantly better. Moreover, the cool component $(T \sim 1.8 \mathrm{keV})$ has a much lower metal abundance than the hot component $(T \sim 8 \mathrm{keV})$, which may be due to the longer cooling time for the cool gas with lower abundance. In addition, it was found that without a main isothermal component, the standard cooling flow model cannot fit the spectrum satisfactorily. From the isothermal model fitting results we also derived the electron density $n_{\mathrm{e}}$, and fitted its radial distribution with a double $-\beta$ model. The $n_{\mathrm{e}}$ profile inferred with the double- $\beta$ model and the deprojected X-ray gas temperature profile were then combined to derive the total mass and the total projected mass of the cluster. The projected mass is lower than that derived from the weak lensing method. However, assuming that the cluster extends to a larger radius $\sim 15^{\prime}$ as found by Clowe \& Schneider (2002), the two results are consistent within the error bars. Furthermore, we calculated the projected mass within the radius of $\sim 153 \mathrm{kpc}$ implied by the presence of a gravitational lensing arc, which is about half of the mass determined from the optical lensing.
\end{abstract}

Key words. galaxies: clusters: individual: Abell 1835 - X-rays: galaxies

\section{Introduction}

There are two yet not very well understood questions concerning clusters of galaxies. The first is: what is the real physical state and process of the X-ray gas in the central region of the cluster? The cooling flow rates in the centers of most galaxy clusters observed recently by XMM-Newton and Chandra are much lower than those predicted by the standard cooling flow model (e.g. A1795, Ettori et al. 2002; Tamura et al. 2001; M 87, Matsushita et al. 2002). An important implication of the low cooling flow rate is that there exist some unknown processes which can heat the gas and thus prevent it from cooling. From the analysis of the X-ray gas, some authors believe that the IntraCluster Medium (ICM) in some clusters is locally isothermal, such as in M 87 (except for the regions associated with the radio structures, Molendi 2002), while some others suggested that the ICM can be better represented by a two-temperature model, in which the cooler component is associated with the Interstellar Medium (ISM) (Makishima et al. 2001). Apparently, a detailed analysis of the spectrum of the ICM in the cluster center is very helpful for exploring the actual physical state and the physical processes in the center. The second is: whether the cluster masses inferred from the two primary observational techniques (optical lensing and X-ray observation) are consistent? For some clusters, the mass determined from the X-ray method is lower than that from the strong gravitational lensing method by a factor $\sim 2$ (e.g. A2218, Loeb \& Mao 1994; A1689, A2163, Miralda-Escudé \& Babul 1995). The reason for this discrepancy may be a prolate ellipsoidal mass distribution or a non-isothermal temperature structure existing in the cluster (Miralda-Escudé \& Babul 1995) or non-thermal pressure supporting the ICM against gravity (e.g., Loeb \& Mao 1994). Interestingly, when a multiphase analysis is adopted, the X-ray and strong lensing masses for the cooling flow clusters present an excellent agreement, but this discrepancy still exists in some other clusters which show no evidence for cooling flows (Allen 1998). However, since the standard multiphase model does not give a satisfactory fit to the new Chandra and XMM-Newton data, it is necessary to explore whether this discrepancy do exist in the cooling flow clusters. Recently, Chen et al. (2003) found a discrepancy of a factor $\sim 2$ in the cooling flow cluster PKS 0745-191 with XMM-Newton observations, while Schmidt et al. (2002) obtained a roughly consistent mass for the two methods with the Chandra data for Abell 1835. However, on larger spatial scales, previous studies have inferred a good agreement between the weak lensing mass and the X-ray mass (e.g. A2218, A2163, Squires et al. 1996, 1997).

Abell 1835 is an important object to study the above properties of the galaxy clusters. It is luminous, with a medium redshift $(z=0.2523)$ and a relaxed structure. ROSAT and $A S C A$ observations show that it has a large cooling flow rate of 
$\sim 1760_{-590}^{+520} M_{\odot} \mathrm{yr}^{-1}$ in its center (Allen et al. 1996). However, recent studies based on XMM-Newton (Turner et al. 2001) and Chandra (Weisskopf et al. 2000) show that the mass deposition rate is not that large. The RGS onboard XMM-Newton has limited the cooling flow rate to $315 M_{\odot} \mathrm{yr}^{-1}$ within a radius of $150 \mathrm{kpc}$ (Peterson et al. 2001), and Chandra derived that the cooling flow rate is $\sim 500 M_{\odot} \mathrm{yr}^{-1}$ within a radius of $250 \mathrm{kpc}$ (Schmidt et al. 2001). Furthermore, the XMM-Newton RGS did not detect any multiphase gas below a certain low temperature $(\sim 2.7 \mathrm{keV})$, inconsistent with the prediction of the standard cooling flow model (Peterson et al. 2001). The gas temperature of Abell 1835 was determined as $\sim 12 \mathrm{keV}$ from the Chandra data (Schmidt et al. 2001), but was determined as $\sim 7.6 \mathrm{keV}$ from the XMM-Newton data (Majerowicz et al. 2002). The discrepancy between the model prediction and observations as well as that between different observations urges a more careful examination with high quality data.

We investigate here the temperature, density, cooling flow rate and mass of Abell 1835 with a deprojection technique based on the data observed by XMM-Newton EPIC. The deprojection technique can reveal the real spectra of the cluster gas in different spherical shells, and we can further determine the deprojected temperature and the mass distribution of the gas in the cluster (e.g., Chen et al. 2003). The XMM-Newton EPIC is the most sensitive $X$-ray telescope which also has high spatial and spectral resolutions, and therefore meets all the requirements for the detailed spectral analysis.

The structure of this paper is as follows: Sect. 2 describes the observation, background correction and the spectral deprojection technique. Section 3 presents the deprojected spectral analysis with three different models: single-temperature model, two-temperature model and cooling flow model. In Sect. 4 we obtain the electron density profile, calculate the total mass, total projected mass and the projected mass within the optical lensing arc, then we discuss the discrepancy between the X-ray mass and the optical lensing mass. Our conclusion is given in Sect. 5 .

Throughout this paper, the energy band is $0.5 \sim 10 \mathrm{keV}$, and unless otherwise noted we use a cosmology with $H_{0}=$ $50 \mathrm{~km} \mathrm{~s}^{-1} \mathrm{Mpc}^{-1}, q_{0}=0.5, \Omega_{\mathrm{m}}=1.0$, and $\Omega_{\Lambda}=0$. Therefore, $1^{\prime}$ corresponds to $296.6 \mathrm{kpc}$ at the distance of Abell 1835 .

\section{Observation and data preparation}

Abell 1835 was observed by XMM-Newton during the phase of performance verification (observation ID is 0098010101). Since MOS1 was operating in Large Window mode, we only use the data coming from MOS2 and pn cameras which were operating in the Full Frame mode and with the thin 1 filter. The total exposure time is $60 \mathrm{ks}$, of which only about $26 \mathrm{ksec}$ is usable for spectroscopic analysis for both pn and MOS2. For the MOS2 data, we use the event with PATTERN $\leq 12$, and for the pn data PATTERN $\leq 4$. The calibration is performed in SAS 5.3.3.

\subsection{Background correction}

Background subtraction was carried out using the same method as that of Majerowicz et al. (2002). The pn data have another source of contamination called out-of-time (OOT) events counted during the read-out (see Strüder et al. 2001). This contamination has also been corrected for in our analysis.

\subsection{Spectral deprojection}

Abell 1835 appears to be a relaxed cluster of galaxies, therefore we assume that the temperature structure of this cluster is spherically symmetric, and apply a deprojection technique as done by Nulsen \& Böhringer (1995). We divide the image of the cluster into 7 annular regions centered on the emission peak for the extraction of spectra and use the outmost ring $\left(8.33^{\prime}-10.42^{\prime}\right)$ to determine the local Cosmic X-ray Background (CXB). However, in the sixth ring the signalto-noise ratio is low, so we only consider the inner five regions $\left(r \leq 6^{\prime}\right)$. The minimum width of the rings was set to $0.75^{\prime}$ which is wide enough to ignore the PSF (Point Spread Function), whose FWHM (Full Width at Half Maximum) is 5" for MOS2 and 6" for pn. For each annular region, an Ancillary Response File (ARF) is generated using SAS, then through the ARF, the vignetting (Arnaud et al. 2001) correction is administered.

Deprojected spectra are calculated by subtracting the contribution from the outer regions for all spectral components. Within each annular region, the spectrum per unit volume is assumed to be the same. The deprojected spectrum of the $i$ th shell is then calculated by subtracting the contributions from $i+1$ th to the outmost shell from the annular spectrum of the corresponding radius (e.g., Matsushita et al. 2002).

\section{Spectral analysis}

\subsection{Single temperature model}

We analysed the deprojected spectra of both MOS2 and pn data using XSPEC version 11.2.0 (Arnaud 1996), and we selected the following model:

$\operatorname{Model}_{1}=\operatorname{Wabs}\left(n_{\mathrm{H}}\right) \times \operatorname{Mekal}(T, z, A, n o r m)$,

here $W a b s$ is a photoelectric absorption model (Morrisson \& McCammon 1983) and Mekal is a single temperature plasma emission model (Mewe et al. 1985, 1986; Kaastra 1992; Liedahl et al. 1995). We fixed the redshift $z$ to 0.2523 and $n_{\mathrm{H}}$ to the Galactic absorption $2.24 \times 10^{20} \mathrm{~cm}^{-2}$ (Dickey \& Lockman 1990). The results are listed in Table 1 and the spectrum of the central region $\left(r<0.75^{\prime}\right)$ for MOS2 data fitted by this single temperature model is shown as Fig. 3a.

To derive the temperature profile of this cluster, we fitted the combined spectra of MOS2 and pn with a single temperature model; the results are also listed in Table 1. From the deprojected temperature profile (the diamonds in Fig. 1), we can see that the temperature is nearly constant in the outer regions but decreases towards the center, which may be connected with 
Table 1. The best-fit free parameters of Abell 1835: the temperature $T$ of MOS2, pn and the combination of them; the abundance $A$ and the normalized constant norm for the combination of MOS2 and pn. norm $=10^{-14} /\left(4 \pi D^{2}\right) \int n_{\mathrm{e}} n_{\mathrm{H}} \mathrm{d} V$, where $D$ is the distance to the source (cm) and $n_{\mathrm{e}}$ is the electron density $\left(\mathrm{cm}^{-3}\right) . L_{\mathrm{X}}$ is the bolometric luminosity and $P$ is the null hypothesis probability of the spectra fitted in XSPEC. The errors represent a confidence level of $90 \%$.

\begin{tabular}{|c|c|c|c|c|c|c|c|}
\hline \multicolumn{2}{|c|}{ Annulus (') } & \multicolumn{3}{|c|}{$T(\mathrm{keV})\left(\chi_{\text {red }}^{2} /\right.$ d.o.f., $\left.P\right)$} & \multirow{2}{*}{$\begin{array}{l}A \text { (solar) } \\
\text { Mos } 2+\text { pn }\end{array}$} & \multirow{2}{*}{$\begin{array}{c}\text { norm }\left(10^{-3} \mathrm{~cm}^{-5}\right) \\
\operatorname{Mos} 2+\mathrm{pn} \\
\end{array}$} & \multirow{2}{*}{$\begin{array}{l}L_{X}\left(10^{45} \mathrm{erg} \mathrm{s}^{-1}\right) \\
(0.001-60 \mathrm{keV})\end{array}$} \\
\hline$r_{1}$ & $r_{2}$ & $\operatorname{mos} 2$ & pn & $\operatorname{mos} 2+\mathrm{pn}$ & & & \\
\hline 0.0 & 0.75 & $\begin{array}{c}6.24 \pm 0.28 \\
(1.31 / 154,0.006)\end{array}$ & $\begin{array}{c}5.46 \pm 0.10 \\
\left(1.56 / 445,3 \times 10^{-13}\right)\end{array}$ & $\begin{array}{c}5.60 \pm 0.10 \\
\left(1.53 / 602,4 \times 10^{-16}\right)\end{array}$ & $0.36 \pm 0.03$ & $9.02 \pm 0.08$ & 3.62 \\
\hline 0.75 & 1.5 & $\begin{array}{c}8.37_{-0.84}^{+0.86} \\
(1.03 / 101,0.395)\end{array}$ & $\begin{array}{c}7.39_{-0.35}^{+0.53} \\
(1.04 / 278,0.293)\end{array}$ & $\begin{array}{c}7.71_{-0.40}^{+0.42} \\
(1.21 / 382,0.003)\end{array}$ & $0.22 \pm 0.07$ & $4.26 \pm 0.07$ & 1.90 \\
\hline 1.5 & 2.25 & $\begin{array}{c}7.82_{-1.28}^{+1.60} \\
(1.10 / 59,0.278)\end{array}$ & $\begin{array}{c}7.16_{-0.59}^{+0.79} \\
(1.01 / 168,0.430)\end{array}$ & $\begin{array}{c}7.27_{-0.51}^{+0.77} \\
(1.04 / 230,0.338)\end{array}$ & $0.23 \pm 0.12$ & $2.45 \pm 0.07$ & 1.07 \\
\hline 2.25 & 3.33 & $\begin{array}{c}7.93_{-1.30}^{+1.89} \\
(1.26 / 45,0.10)\end{array}$ & $\begin{array}{c}7.10_{-0.95}^{+1.20} \\
(0.84 / 108,0.885)\end{array}$ & $\begin{array}{c}7.33_{-0.76}^{+1.05} \\
(1.18 / 156,0.06)\end{array}$ & $0.35 \pm 0.18$ & $1.55 \pm 0.07$ & 0.70 \\
\hline 3.33 & 6.0 & $\begin{array}{c}7.32_{-2.84}^{+7.31} \\
(0.84 / 27,0.80)\end{array}$ & $\begin{array}{c}6.61_{-1.31}^{+1.76} \\
(1.26 / 116,0.03)\end{array}$ & $\begin{array}{c}6.63_{-1.20}^{+1.52} \\
(1.37 / 146,0.002) \\
\end{array}$ & $0.36_{-0.32}^{+0.39}$ & $1.42 \pm 0.12$ & 0.61 \\
\hline
\end{tabular}

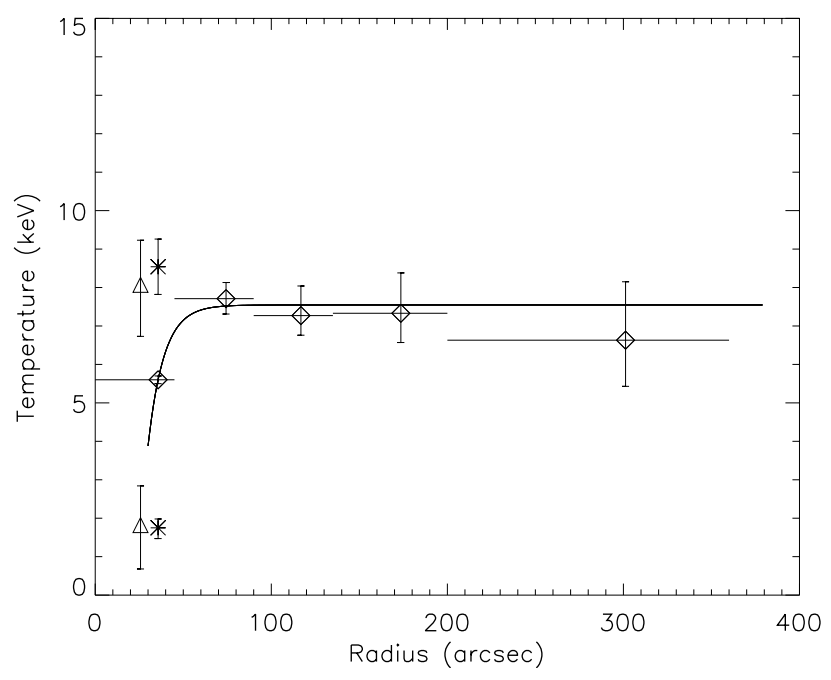

Fig. 1. Temperature profile of Abell 1835 for the combination of mos2 and pn with a confidence level of $90 \%$. (Diamonds: temperature from the 1T model fitting of the combined spectra; stars: temperature from the 2T model fitting of the pn central spectrum; triangles: temperature from the $2 \mathrm{~T}$ model fitting of the MOS2 central spectrum. We have offset the triangles $10^{\prime \prime}$ to the left so as to illustrate the two fits clearly.) The solid line is the best-fitted profile of the $1 \mathrm{~T}$ model.

the gas cooling. Then we fit the temperature profile with the formula:

$T(r)=a \mathrm{e}^{b r}+c$.

The best fit parameters are: $a=-101.9 \mathrm{keV}, b=$ $-0.11 \operatorname{arcsec}^{-1}, c=7.55 \mathrm{keV}$.

Our result does not differ much from that of Majerowicz et al. (2002) based on the same data, although what Majerowicz et al. derived is the projected temperature. However, the deprojected temperature from the Chandra data (Schmidt et al. 2001), $\sim 12 \mathrm{keV}$, is much higher than our result. This may be due to the difficulty in identifying the background flares in the Chandra data (Markevitch 2002).

\subsection{Double temperature model}

From the null hypothesis probability $P$ (the adopted level of a fit is $P \geq 0.1$, and when $0.01 \leq P<0.1$, the fit is marginally acceptable) in Table 1 we find that the spectral fitting with the single-temperature model is acceptable for the outer regions, but unacceptable for the central region. Since the calibrations of pn and MOS2 are not quite consistent with each other, we fit the central spectrum of pn and MOS2 with the two-temperature model respectively:

$$
\begin{aligned}
& \text { Model }_{2}=\operatorname{Wabs}\left(n_{\mathrm{H}}\right) \times\left(\operatorname{Mekal}\left(T_{1}, z, A_{1}, \text { norm }_{1}\right)\right. \\
& \left.+\operatorname{Mekal}\left(T_{2}, z, A_{2}, \operatorname{norm}_{2}\right)\right) \text {, }
\end{aligned}
$$

which means that there are two components in this region with different temperatures, and the free parameters have the same meaning as in Sect. 3.1. Assuming that the two components have the same abundances, we fix the abundances of the cool component to those of the hot gas (see Fig. 3b for MOS2), and obtain the best-fit parameters listed in Table 2. Note that the cool component only has a small volume filling fraction of less than a few percent for both pn and MOS2 (see $f_{\text {vol }}$ in Table 2). Although the best parameters derived from pn and MOS2 do not agree with each other in detail, the values of $\chi^{2}$ for both of them improved significantly and are marginally acceptable.

We further leave the abundances free and obtain a even better fit (see Fig. 3c). The resultant model parameters of pn and MOS2 are now consistent with each other, as shown in Table 3, where $F$ is derived from a simple $F$-test (Bevington 1969) and indicates the degree of confidence of the model in which both abundances are left free parameters compared to the model setting the two abundances the same. From $F$ we know that the substitution of this model for pn data is significant with $>99.9 \%$ confidence, while for MOS2 it is about $80 \%$. The existence of the two abundance components implies that the distribution of the abundance for both temperature components is inhomogeneous in the central region of the cluster. As shown in Table 3, in the steady cooling flow scenario, there exist two temperature components, and the cool one with a temperature less than 
Table 2. The best-fit parameters of Abell 1835 using a two-temperature model for the central region $\left(0.0^{\prime}-0.75^{\prime}\right)$ and assuming that the two components have the same abundance. The error bars are at the $90 \%$ confidence level. $f_{\text {vol }}$ is the volume fraction of the cool component.

\begin{tabular}{|c|c|c|c|c|c|c|c|c|}
\hline \multirow[t]{2}{*}{ Detector } & \multicolumn{2}{|c|}{$T(\mathrm{keV})$} & \multirow{2}{*}{$\begin{array}{c}A \\
\text { (solar) }\end{array}$} & \multicolumn{2}{|c|}{ norm $\left(10^{-3} \mathrm{~cm}^{-5}\right)$} & \multirow[t]{2}{*}{$f_{\mathrm{vol}}$} & \multirow[t]{2}{*}{$\chi_{\text {red }}^{2} /$ d.o.f. } & \multirow[t]{2}{*}{$P$} \\
\hline & $T_{1}$ & $T_{2}$ & & norm $_{1}$ & norm $_{2}$ & & & \\
\hline pn & $8.17_{-0.77}^{+1.01}$ & $2.02 \pm 0.28$ & $0.35 \pm 0.05$ & $6.67_{-0.71}^{+0.60}$ & $2.59_{-0.64}^{+0.73}$ & 0.02 & $1.21 / 443$ & 0.02 \\
\hline MOS2 & $6.60 \pm 0.33$ & $0.71 \pm 0.18$ & $0.44 \pm 0.07$ & $8.67_{-0.17}^{+0.10}$ & $0.16 \pm 0.08$ & 0.0002 & $1.24 / 152$ & 0.02 \\
\hline Combined & $8.05_{-0.70}^{+0.82}$ & $2.12_{-0.34}^{+0.33}$ & $0.37 \pm 0.04$ & $6.84_{-0.66}^{+0.63}$ & $2.35_{-0.65}^{+0.67}$ & 0.02 & $1.29 / 600$ & $2 \times 10^{-6}$ \\
\hline
\end{tabular}

Table 3. The best-fit parameters of Abell 1835 using a two-temperature model for the central region $\left(0.0^{\prime}-0.75^{\prime}\right)$, but leaving the abundances of the two components free. The error bars are at the $90 \%$ confidence level. $F$ is the confidence level of using this $2 \mathrm{~T} 2 \mathrm{~A}$ model instead of the 2T1A model.

\begin{tabular}{|c|c|c|c|c|c|c|c|c|c|}
\hline \multirow[t]{2}{*}{ Detector } & \multicolumn{2}{|c|}{$T(\mathrm{keV})$} & \multicolumn{2}{|c|}{$A$ (solar) } & \multicolumn{2}{|c|}{$\operatorname{norm}\left(10^{-3} \mathrm{~cm}^{-5}\right)$} & \multirow[t]{2}{*}{$\chi_{\text {red }}^{2} /$ d.o.f. } & \multirow[t]{2}{*}{$P$} & \multirow[t]{2}{*}{$F$} \\
\hline & $T_{1}$ & $T_{2}$ & $A_{1}$ & $A_{2}$ & norm $_{1}$ & norm $_{2}$ & & & \\
\hline pn & $8.54 \pm 0.72$ & $1.75_{-0.28}^{+0.23}$ & $0.50 \pm 0.08$ & $0.13 \pm 0.06$ & $6.39_{-0.50}^{+0.55}$ & $3.34 \pm 0.50$ & $1.13 / 442$ & 0.03 & $>0.999$ \\
\hline MOS2 & $8.07_{-1.34}^{+1.16}$ & $1.82_{-1.14}^{+1.02}$ & $0.52_{-0.11}^{+0.14}$ & $0.10_{-0.10}^{+0.21}$ & $7.36_{-1.17}^{+1.30}$ & $2.08_{-1.76}^{+0.98}$ & $1.23 / 151$ & 0.03 & 0.80 \\
\hline Combined & $8.32_{-0.65}^{+0.60}$ & $1.75_{-0.31}^{+0.23}$ & $0.49 \pm 0.07$ & $0.12 \pm 0.05$ & $6.69_{-0.44}^{+0.52}$ & $3.0_{-0.48}^{+0.44}$ & $1.22 / 599$ & 0.0001 & $>0.999$ \\
\hline
\end{tabular}

$2 \mathrm{keV}$ has a lower abundance. This may be due to the metal-rich gas cooling together with the metal-poor gas by bremsstrahlung emission until $\sim 2 \mathrm{keV}$, below which line cooling becomes important, and the metal-rich gas would cool at a much faster rate than the metal-poor gas (Fabian et al. 2001). So in the steady cooling flow scenario the metal-rich component cannot be seen at temperatures below about $2 \mathrm{keV}$ and thus the cool component presents a lower abundance.

Although the combined spectra of pn and MOS2 are not suitable for the two-temperature model fitting because of the slight inconsistence between their calibrations, we still perform the combined fits to calculate the mass in the $2 \mathrm{~T}$ model method easily (see Sect. 4.4). The resulting parameters as listed in Tables 2 and 3 lie between the two sets of parameters derived separately from the pn and MOS2 data.

We also fit the outer spectra with the two-temperature model, but the fits do not improve significantly.

From the analyses above, we know that a two-temperature model may represent the spectrum of the central region much better, which is possibly due to the existence of cooling gas in the cluster center or the possible presence of ISM associated with the cD galaxy (Makishima et al. 2001). However, for the outer parts, the fit of the single-temperature model is good enough to be acceptable. So we inferred that in the cluster center there exist two gas components with different temperatures, while in the outer regions the gas is isothermal (Kaastra et al. 2003).

Figure 2 is the deprojected abundance profile. It can be seen that the abundance is higher in the cluster center and tends to constant in the outer region. This indicates that the excess metal in the cluster center is produced in the $\mathrm{cD}$ galaxy and ejected into the ICM (Makishima et al. 2001).

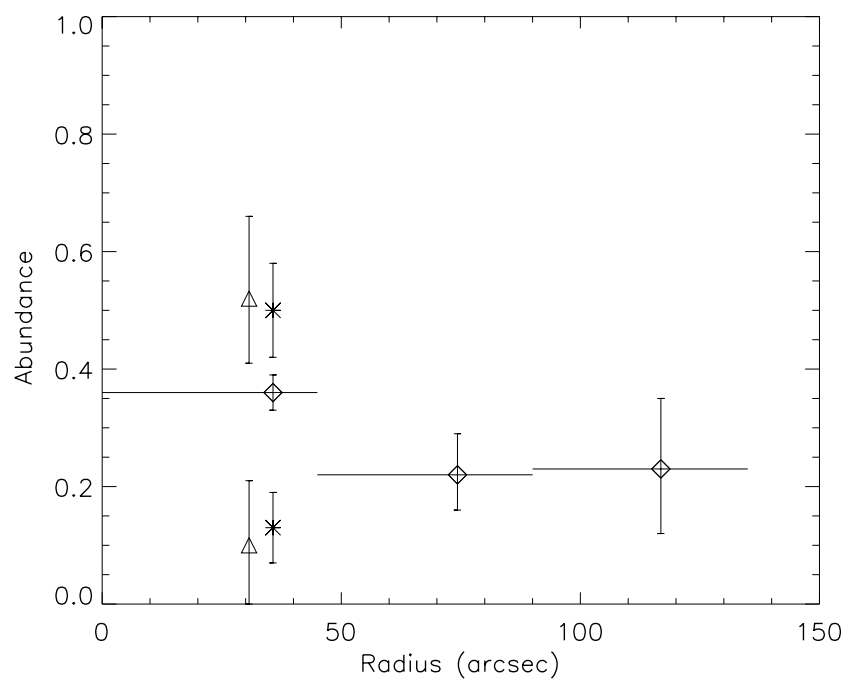

Fig. 2. Abundance profile of Abell 1835 in units of solar metallicity with a confidence level of $90 \%$. The symbols have the same meaning as Fig. 1.

\subsection{Asorbing column density}

To verify if the observed $n_{\mathrm{H}}$ is consistent with the Galactic column density, we fit the spectra with the parameter $n_{\mathrm{H}}$ left free. First, we perform joint fits to 5 pn and MOS2 spectra respectively with a single temperature model, assuming that the 5 spectra have the same $n_{\mathrm{H}}$, and the results are shown in the upper part of Table 4. It can be seen that the two best-fit $n_{\mathrm{H}}$ are much smaller than the Galactic column density $\sim 2.24 \times 10^{20} \mathrm{~cm}^{-2}$. Since the central spectrum can be fitted well by a two-temperature model, we consider a joint fit with a two-temperature model for the central spectrum and a single temperature model for the outer 4 spectra, and the hydrogen 
column density $n_{\mathrm{H}}$ of each spectrum is assumed to be the same. The results are listed in the lower part of Table 4 , where $F$ is the confidence level corresponding to using the $2 \mathrm{~T}$ model to fit the central spectrum instead of using the 1T model to fit all five spectra. We find that the fits become better, and from $F$ we know that the substitution by a $2 \mathrm{~T}$ model is necessary. It is also shown in Table 4 that the $n_{\mathrm{H}}$ determined from the pn spectra is still smaller and about half the Galactic column density, while the $n_{\mathrm{H}}$ obtained from the MOS2 spectra is consistent with the Galactic column density. We should note that for many clusters the best-fit column density derived from the MOS is always in good agreement with the Galactic value, while that estimated from pn data is systematically smaller (Molendi \& Pizzolato 2001). This may be due to the MOS detector having a more reliable calibration than the pn detector.

We also note here that the joint fit with a two-temperature model for the central spectrum can yield a much more believable $n_{\mathrm{H}}$, and this is also evidence for the existence of two gas components with different temperatures in the cluster center.

Through the analysis above, we can conclude that the observed $n_{\mathrm{H}}$ of XMM-Newton is consistent with the Galactic column density, and so the analysis in Sects. 3.1 and 3.2 is reliable. In addition, the MOS data are probably much better for determining $n_{\mathrm{H}}$ than the pn data.

\subsection{Cooling flow model}

X-ray observations of clusters of galaxies show that in the central regions of some clusters the cooling time of the ICM is significantly less than the Hubble time (Edge et al. 1992; White et al. 1997; Peres et al. 1998). ROSAT and ASCA observations show that Abell 1835 is the cluster that contains the largest cooling flow rate (Allen et al. 1996). Here we use the MOS2 data, which are assumed to be better for the determination of $n_{\mathrm{H}}$ and thus the cooling flow rate (Molendi \& Pizzolato 2001), to calculate the cooling flow rate of Abell 1835. There are two different methods for calculating the cooling flow rate: spectral method and spatial method.

Since we have already deprojected the spectrum, i.e., the contribution of the ambient gas has been excluded, the spectrum should be fitted well with the standard cooling flow model:

$$
\operatorname{Model}_{3}=\operatorname{Wabs}\left(n_{\mathrm{H}}\right) \times\left(\operatorname{Zwabs}\left(\Delta n_{\mathrm{H}}\right) \times \operatorname{Mkcflow}(\dot{M})\right),
$$

where Wabs was described in Sect. 3.1, Zwabs is an intrinsic photoelectric absorption model (Morrison \& McCammon 1983), and Mkcflow is a cooling flow model (Fabian 1988); $\Delta n_{\mathrm{H}}$ is the intrinsic absorption and $\dot{M}$ the rate of gas cooling out of the flow. Since the cooling flow radius of Abell 1835 is $230 \mathrm{kpc}$ (Allen 2000) at which the cooling time first exceeds the age of the universe, we only fit the central spectrum $(\sim 225 \mathrm{kpc})$ with this model (Table 5). It can be seen that to fit the spectrum a cut-off temperature, low $T$, is needed, which is found to be $2.3 \mathrm{keV}$. If we fix low $T=0.01 \mathrm{keV}$, the $\chi^{2}$ value will be much larger (see Table 5) and from $P$ we know that this fit is unacceptable. Therefore, the standard cooling flow model cannot fit the spectrum satisfactorily.
Table 4. The absorbing column density determined by pn and MOS2 spectra assuming that all the spectra have the same $n_{\mathrm{H}}$. $1 \mathrm{~T}$ model: the joint fit with a single temperature model. $2 \mathrm{~T}+1 \mathrm{~T}$ model: the joint fit with a two-temperature model for the central spectrum and a single temperature model for the outer 4 spectra, where $F$ is the confidence level of the substitution by the $2 \mathrm{~T}+1 \mathrm{~T}$ model.

\begin{tabular}{cccccc}
\hline \hline Model & Detector & $\begin{array}{c}n_{\mathrm{H}} \\
\left(10^{20} \mathrm{~cm}^{-2}\right)\end{array}$ & $\chi_{\text {red }}^{2} /$ d.o.f. & $P$ & $F$ \\
\hline $1 \mathrm{~T}$ & pn & $0.01_{-0.01}^{+0.30}$ & $1.15 / 1114$ & 0.0004 & \\
& MOS2 & $1.05_{-0.62}^{+0.65}$ & $1.15 / 385$ & 0.02 & \\
& & & & & \\
$2 \mathrm{~T}+1 \mathrm{~T}$ & pn & $0.99_{-0.43}^{+0.45}$ & $1.06 / 1111$ & 0.08 & $>0.999$ \\
& MOS2 & $1.97_{-0.98}^{+1.12}$ & $1.13 / 382$ & 0.035 & $>0.90$ \\
\hline
\end{tabular}

When adding an isothermal Mekal component, the fit becomes much better, as shown in Table 6. $F$ shows that the replacement $(($ Mekal + Mkcflow) instead of Mkcflow) is significant, i.e., the cooling flow model with a Mekal component is more reliable, which implies that there should exist an isothermal component in the cluster. Then, the cooling flow rate $\dot{M}$ is about $656.2_{-360.2}^{+403.4} M_{\odot} \mathrm{yr}^{-1}$ within $225 \mathrm{kpc}\left(0.75^{\prime}\right)$. The spectrum of the central region $\left(0.0^{\prime}-0.75^{\prime}\right)$ fitted by this model for MOS2 data is shown in Fig. $3 \mathrm{~d}$.

It can also be seen from Table 6 that the intrinsic absorption $\Delta n_{\mathrm{H}}$ of Abell 1835 is very small and consistent with zero within the error bars, which is in agreement with the previous results, such as those of RGS (Peterson et al. 2001) and Molendi \& Pizzolato (2001) in which the projected spectra are used. If we adopt the best-fit result from the analysis in Schmidt et al. (2001) of the Chandra dataset, and fix $\Delta n_{\mathrm{H}}=0.25 \times 10^{22} \mathrm{~cm}^{-2}$, we measure $\dot{M}=262.5_{-262.5}^{+169.5} M_{\odot} \mathrm{yr}^{-1}$, which is unacceptable at the adopted level of $P=0.01$. So the intrinsic absorption of Abell 1835 is very small and close to zero.

Another method for calculating the cooling flow rate is the spatial method. According to the energy conservation, this method can be expressed as (White et al. 1997):

$$
\begin{aligned}
L_{x}(i)= & \dot{M}(i)[h(i)+f(i) \triangle \phi(i)] \\
& +\sum_{i^{\prime}=1}^{i^{\prime}=i-1} \dot{M}\left(i^{\prime}\right)[\Delta h(i)+\Delta \phi(i)],
\end{aligned}
$$

where $L_{x}$ is the bolometric luminosity, shown in Table $1 ; \dot{M}(i)$ is the mass deposition in shell $i ; \sum_{i^{\prime}=1}^{i^{\prime}=1-1} \dot{M}\left(i^{\prime}\right)$ is the mass of gas that needs to pass through shell $i$ to give rise to the radiation and mass deposition in interior shells; $\Delta \phi(i)$ is the change in the gravitational potential; $h(i)=\frac{5}{2} k T(i) / \mu m_{\mathrm{p}}$ is the temperature in units of energy per particle mass of the hot gas; $f(i)$ is a fraction of the overall change in the cluster potential, and in this analysis $f(i)=1$ is used. The first two terms in this equation represent the mass that is left in shell $i$, and the second two terms represent the mass that flows through to the next interior shell. From this method, we can estimate the cooling flow rate within the central region $(r<225 \mathrm{kpc}) \sim$ about $1600 M_{\odot} \mathrm{yr}^{-1}$, consistent with the previous result of Chandra from the same method (Schmidt et al. 2001). 
Table 5. The best-fit parameters for the central region $\left(r<0.75^{\prime}\right)$ of MOS2 data by the standard cooling flow model. The errors are at the $90 \%$ confidence level. $\dot{M}$ is the mass deposition rate, and $\Delta n_{\mathrm{H}}$ is the intrinsic absorption.

\begin{tabular}{ccccccc}
\hline \hline $\begin{array}{c}\text { low } T_{\text {cf }} \\
(\mathrm{keV})\end{array}$ & $\begin{array}{c}\text { high } T_{\text {cf }} \\
(\mathrm{keV})\end{array}$ & $\begin{array}{c}A \\
(\text { solar })\end{array}$ & $\begin{array}{c}\dot{M} \\
\left(M_{\odot}\right)\end{array}$ & $\begin{array}{c}\Delta n_{\mathrm{H}} \\
\left(10^{22} \mathrm{~cm}^{-2}\right)\end{array}$ & $\chi_{\text {red }}^{2} /$ d.o.f. & $P$ \\
\hline $2.27_{-0.58}^{+0.84}$ & $13.75_{-2.62}^{+2.76}$ & $0.46 \pm 0.08$ & $2527.4_{-488.7}^{+967.9}$ & $0.0_{-0.0}^{+0.003}$ & $1.25 / 152$ & 0.02 \\
$0.01(\mathrm{fix})$ & $18.21_{-1.31}^{+1.88}$ & $0.35 \pm 0.08$ & $1766.6_{-141.0}^{+118.7}$ & $0.06_{-0.01}^{+0.02}$ & $1.37 / 153$ & 0.001 \\
\hline
\end{tabular}

Table 6. The same as Table 5 but for the cooling flow model with a Mekal component. $F$ is the confidence level when using ( $M e k a l+M k c f l o w)$ instead of the standard cooling flow model.

\begin{tabular}{cccccccccc}
\hline \hline $\begin{array}{c}T_{\text {mekal }} \\
(\mathrm{keV})\end{array}$ & $\begin{array}{c}\text { low } T_{\text {cf }} \\
(\mathrm{keV})\end{array}$ & $\begin{array}{c}\text { high } T_{\text {cf }} \\
(\mathrm{keV})\end{array}$ & $\begin{array}{c}A \\
(\text { solar })\end{array}$ & $\begin{array}{c}\text { norm } \\
\left(10^{-3} \mathrm{~cm}^{-5}\right)\end{array}$ & $\begin{array}{c}\dot{M} \\
\left(M_{\odot}\right)\end{array}$ & $\begin{array}{c}\Delta n_{\mathrm{H}} \\
\left(10^{22} \mathrm{~cm}^{-2}\right)\end{array}$ & $\chi_{\text {red }}^{2} /$ d.o.f. & $P$ & $F$ \\
\hline $7.30_{-0.60}^{+0.91}$ & $0.01(\mathrm{fix})$ & $=T_{\text {mekal }}$ & $0.44 \pm 0.08$ & $7.20_{-1.13}^{+0.92}$ & $656.2_{-360.2}^{+403.4}$ & $0.06 \pm 0.06$ & $1.22 / 152$ & 0.03 & $>0.999$ \\
$6.38_{-0.38}^{+0.36}$ & 0.01 (fix) & $=T_{\text {mekal }}$ & $0.43 \pm 0.07$ & $8.41_{-0.85}^{+0.63}$ & $262.5_{-262.5}^{+169.5}$ & 0.25 (fix) & $1.31 / 153$ & 0.006 & \\
\hline
\end{tabular}

The result derived from the spatial method gives the total loss rate of the energy, but the cooling flow rate obtained from the spectral method only contains the mass deposition rate during the cooling flow. The discrepancy between these two methods implies that there should exist some other energy sources, for example heating by AGN (see e.g., Böhringer et al. 2002) or thermal conduction (e.g. Voigt et al. 2002) to support the X-ray emission in the center of the cluster.

With any acceptable model (the two-temperature model or the cooling flow model with a mekal component) we have investigated above, we can conclude that in the cluster center there should always exist a locally isothermal component and there should also exist a minor component which may be a single phased cool component or a multiphased one. However, with the current data we can not discriminate between these two models.

\section{Mass analysis}

\subsection{Electron density}

Here, we have divided the cluster into 17 annular regions centered on the emission peak. By the deprojection technique we can calculate the photon counts in each corresponding shell. Then, using the abundance and the temperature profile, the normalized constant of each region, norm, can be estimated. Furthermore, since:

norm $=10^{-14} /\left(4 \pi D^{2}\right) \int n_{\mathrm{e}} n_{\mathrm{H}} \mathrm{d} V$,

we can derive the deprojected electron density $n_{\mathrm{e}}$, shown as the triangles in Fig. 4.

We fit the electron density with both single- $\beta$ and double$\beta$ model. First, we fit it with a single- $\beta$ model (e.g., Cavaliere $\&$ Fusco-Femiano 1976) in which the electron density profile $n_{\mathrm{e}}(r)$ is defined as:

$n_{\mathrm{e}}(r)=n_{0}\left[1+\left(\frac{r}{r_{\mathrm{c}}}\right)^{2}\right]^{-\frac{3}{2} \beta}$,

where $n_{0}$ is the central density, $r_{\mathrm{c}}$ is the core radius, and $\beta$ is the slope. The best-fit parameters are: $n_{0}=0.06 \mathrm{~cm}^{-3}, r_{\mathrm{c}}=0.21 \operatorname{arcmin}, \beta=0.56, \chi^{2}=60.33$, d.o.f. $=14$ and the best-fit single- $\beta$ model profile is plotted as a dotted line in Fig. 4. We can see that it does not fit well, especially in the outer regions. For this reason we fit it with a “double- $\beta$ " model (see Chen et al. 2003):

$n_{\mathrm{e}}(r)=n_{01}\left[1+\left(\frac{r}{r_{c 1}}\right)^{2}\right]^{-\frac{3}{2} \beta_{1}}+n_{02}\left[1+\left(\frac{r}{r_{c 2}}\right)^{2}\right]^{-\frac{3}{2} \beta_{2}}$.

Note that this model fits the electron density directly and is different from the common double- $\beta$ model that was used to fit the surface brightness. The best fit parameters are: $n_{01}=$ $0.003 \mathrm{~cm}^{-3}, r_{c 1}=1.84 \operatorname{arcmin}, \beta_{1}=1.24, n_{02}=0.08 \mathrm{~cm}^{-3}$, $r_{c 2}=0.155$ arcmin, $\beta_{2}=0.60, \chi^{2}=12.25$, d.o.f. $=11$ and the best-fit line is shown as the solid line in Fig. 4. It can be seen from the $\chi^{2}$ value that the electron density profile is fitted better by a double- $\beta$ model than by a single- $\beta$ model, so in the following calculations we use the electron density profile inferred from the double- $\beta$ model.

\subsection{Total mass}

Assuming spherical symmetry and hydrostatic equilibrium, the total mass profile can be determined when the radial profiles of the gas density and temperature are known. We calculate the gravitational mass of Abell 1835 with the hydrostatic equation (Fabricant et al. 1980):

$M_{\mathrm{tot}}(<r)=-\frac{k_{\mathrm{B}} T r^{2}}{G \mu m_{\mathrm{p}}}\left[\frac{\mathrm{d}\left(\ln n_{\mathrm{e}}\right)}{\mathrm{d} r}+\frac{\mathrm{d}(\ln T)}{\mathrm{d} r}\right]$,

here $k_{\mathrm{B}}$ is the Boltzmann constant, $G$ is the gravitational constant, $\mu$ is the mean molecular weight of the gas in units of $m_{\mathrm{p}}$, the proton mass. For a fully ionized gas with a standard cosmic abundance, a suitable value is $\mu=0.6$.

Using the best-fit profiles of the electron density $n_{\mathrm{e}}(r)$ and the deprojected temperature $T(r)$ we can obtain the total mass profile shown in Fig. 5. It was found that the total mass $M_{\text {tot }}$ within the radius of $6^{\prime}$ is $1.05 \pm 0.13 \times 10^{15} M_{\odot}$, which is consistent with that found by Majerowicz et al. (2002) within the errors. 

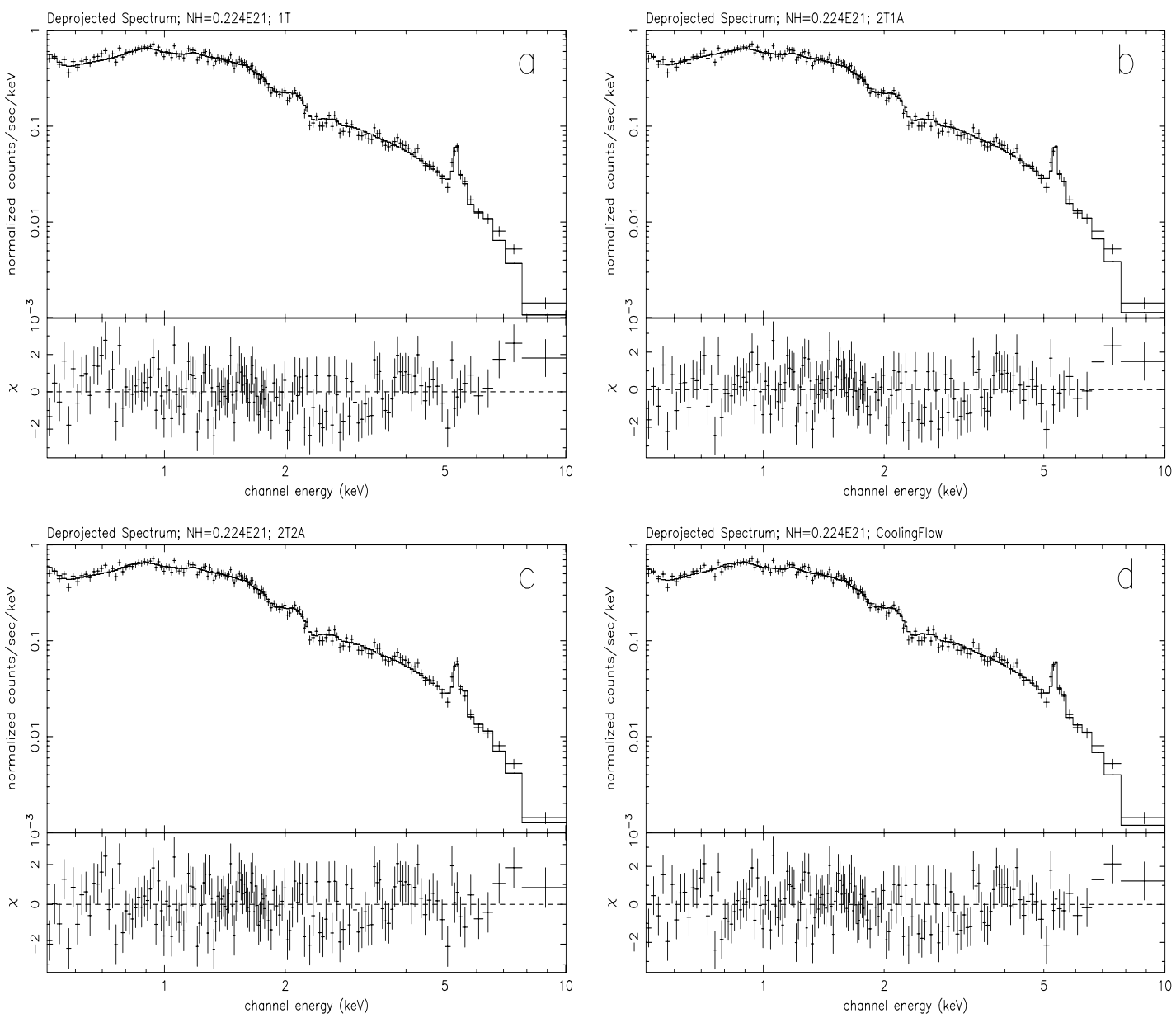

Fig. 3. The spectrum of the central region $\left(r<0.75^{\prime}\right)$ for MOS2 data of Abell 1835. a) Fitted by the 1T model; b) fitted by the 2T1A model; c) fitted by the 2T2A model; d) fitted by the cooling flow model (wabs (mekal + zwabs (mkcflow))).

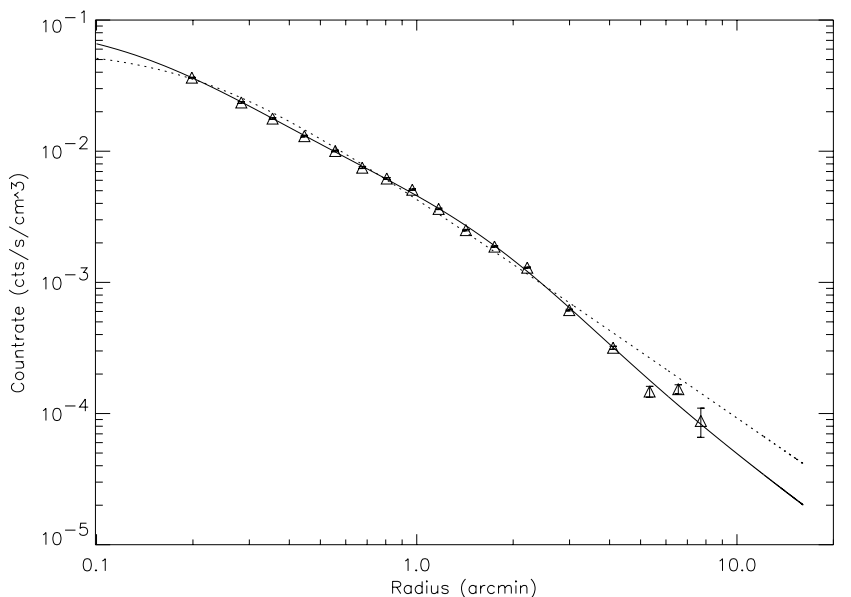

Fig. 4. Electron density profile of Abell 1835 . The error bars are at a $68 \%$ confidence level. The solid line is the best-fit profile for a double- $\beta$ model, and the dotted line for a single $-\beta$ model.

\subsection{Total projected mass}

We calculate the projected mass profile of Abell 1835 for another universe model: $H_{0}=100 \mathrm{~km} \mathrm{~s}^{-1} \mathrm{Mpc}^{-1}, \Omega_{\mathrm{m}}=0.3$, and $\Omega_{\Lambda}=0.7$ and compare it with that of Clowe \& Schneider (2002), who also calculated the total mass in the same

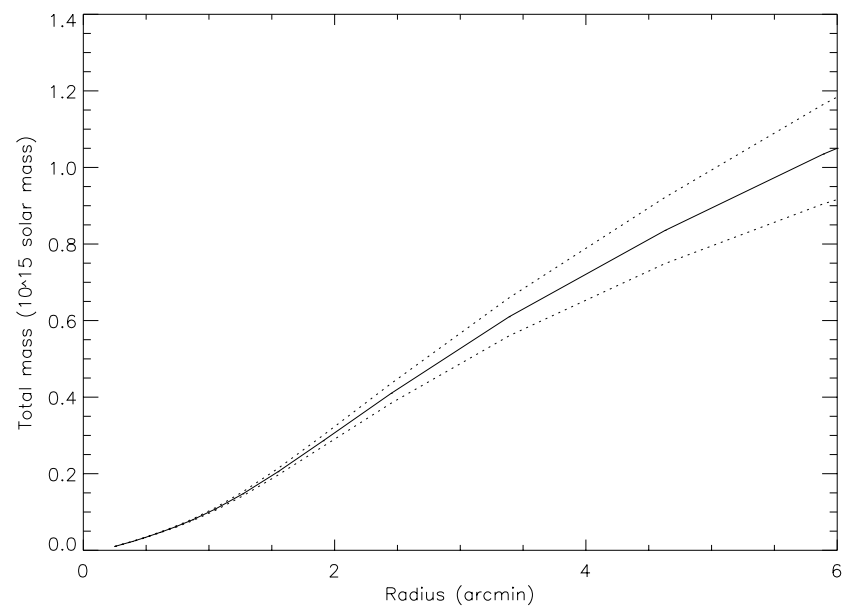

Fig. 5. The total mass profile of Abell 1835. The error bars (dotted lines) are at a $68 \%$ confidence level.

universe model using the weak lensing method based on the data from the ESO/MPG Wide Field Imager (see Fig. 6). The upper panel shows our projected mass within $6^{\prime}$, and it can be seen that the two results are consistent within our error bars in the radial range between $1^{\prime}$ and $4^{\prime}$, where lensing mass estimates are available. By increasing the radius, our mass 

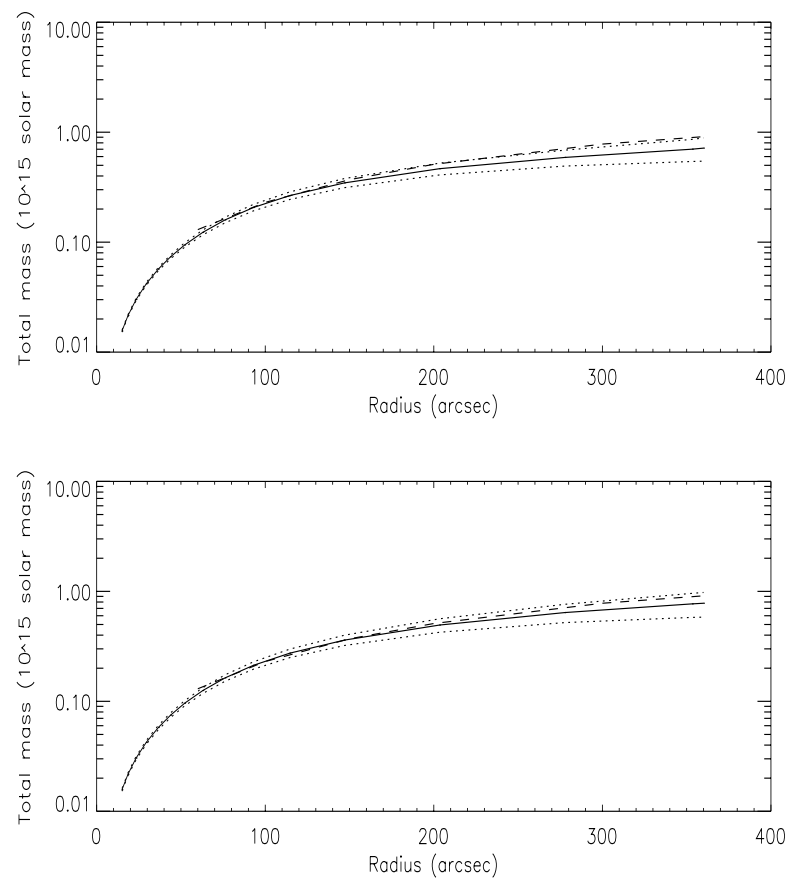

Fig. 6. The projected mass profile in the universe model: $H_{0}=$ $100 \mathrm{~km} \mathrm{~s}^{-1} \mathrm{Mpc}^{-1}, \Omega_{\mathrm{m}}=0.3, \Omega_{\Lambda}=0.7$ (shown as the solid line; the dotted lines mark the confidence level of $68 \%$ ), and compared with that of Clowe \& Schneider (shown as the dashed line). In the upper panel, the solid line presents the projected mass within 6'; the lower panel is for the projected mass within $15^{\prime}$.

measurement becomes lower than that of Clowe \& Schneider. Since Clowe \& Schneider have derived the lensing mass profile up to $15^{\prime}$, we also extrapolate our best-fit temperature and electron density profiles to that large radius, then calculate the projected mass, shown in the lower panel. The two results are consistent within our error bars. Moreover, if we assume that the mass in the outer regions is distributed as derived from Clowe \& Schneider, we estimate a contribution projected from $r>6^{\prime}$ to the inner part of about $0.16 \times 10^{15} M_{\odot}$. By considering this contribution, the two results in the upper panel will also be consistent. Therefore we conclude that the X-ray mass shows an excellent agreement with the weak lensing mass at large radii.

\subsection{Mass within the optical lensing arc}

The optical observation (taken on 27 February 1998 with the Canada France Hawaii Telescope, CFHT) shows an arc $\sim 153 \mathrm{kpc}$ from the center of Abell 1835 which is thought to be a gravitationally lensed image of a galaxy far behind Abell 1835 (see Fig. 2 of Schmidt et al. 2001). We estimate the X-ray projected mass inside this arc: $M_{\text {arc }}=0.80 \pm 0.02 \times$ $10^{14} M_{\odot}$ (errors are at $68 \%$ confidence level).

Since there is a two-temperature structure in the central part, we also calculate the projected mass in the $2 \mathrm{~T}$ model: assuming that the two temperature components are in pressure equilibrium. Through the two-temperature model fitting, we obtained the temperature and norm for each component. Then using Eq. (6) the electron density $n_{\mathrm{e}}$ of each can be derived. Because of the pressure equilibrium, we can calculate the projected mass from any component by using Eq. (9), and the result is about $0.7 \times 10^{14} M_{\odot}$, which is still in agreement with that from the $1 \mathrm{~T}$ model.

Allen et al. (1996) calculated the lensing mass inside the radius of this arc, and the result is between $1.4 \times 10^{14} M_{\odot}$ and $2 \times 10^{14} M_{\odot}$ for the redshift of the arc $0.6<z_{\text {arc }}<3.0$. Schmidt et al. (2001) also calculate the X-ray projected mass inside this arc using the Chandra data and their value is $1.06 \sim 1.27 \times$ $10^{14} M_{\odot}$. Both results are larger than ours. We will discuss the reasons in the following section.

\subsection{The discrepancy between the $X$-ray mass and the optical lensing mass}

Section 4.4 shows that the projected mass within the optical lensing arc we calculated is not consistent with previous results. Our estimate is lower than that derived from the analysis of Chandra data in Schmidt et al. (2001), the main reason being probably the large difference of the temperatures determined by XMM-Newton and Chandra, which directly causes the difference of the mass. The X-ray gas temperature determined by Chandra is about $12 \mathrm{keV}$, but it is only about $7.6 \mathrm{keV}$ for XMM-Newton.

On the other hand, Bartelmann (1995) shows that on average the cluster mass required for large arcs will be lower by a factor of $\sim 1.6$ than expected from radially symmetric models, and that there is a probability of $\sim 20 \%$ of overestimating the actual cluster mass by a factor of $\sim 2$. The inner part of the $\mathrm{X}$-ray isocontours of Abell 1835 is elliptical with an axis ratio of $f=0.85$ (see Fig. 4 in Schmidt et al. 2001). Therefore, one reason for the difference between our result and the lensing mass might be the assumption of a symmetric model when calculating the lensing mass. Another reason may be the existence of radio structure (Ivison et al. 2000) in the center region of Abell 1835 like in PKS 0745-191 (Chen et al. 2003), although the radio emission of the latter is much stronger. The radio plasma may fill a larger volume in the central region of the cluster or provide some additional pressure to support the $\mathrm{X}$-ray gas. Therefore, when we consider the radio component, the projected mass may be larger and may be consistent with that of the optical lensing.

\section{Conclusion}

We have presented a detailed analysis of about $26 \mathrm{ks}$ of XMM-Newton observations of the galaxy cluster Abell 1835 . Through the deprojected spectra analysis we derived the deprojected temperature profile, which is flat in the outer regions but decreases towards the center. The abundance is higher in the cluster center, which may be caused by the cD galaxy (Makishima et al. 2001). We also fit the spectrum in the central region with a two-temperature model, and the fit becomes better. It was found that the two temperature components coexist in the cluster center which is due to the gas cooling and/or the ISM associated with the $\mathrm{cD}$ galaxy (Makishima et al. 2001). The lower temperature component also has a lower abundance, which may be due to the fact that the metal-rich gas cools faster when temperature falls below about $2 \mathrm{keV}$ 
(Fabian et al. 2001). Then considering a cooling flow model, we find that the intrinsic absorption of Abell 1835 is very small if not zero and the cooling flow rate is $656.2_{-360.2}^{+403.4} M_{\odot} \mathrm{yr}^{-1}$, which is much smaller than that derived by the spatial method of $\sim 1600 M_{\odot} \mathrm{yr}^{-1}$. This implies that there should exist some other energy sources that can heat the gas and prevent it from cooling down. Furthermore, we showed that the cooling flow model without a Mekal component cannot explain the data satisfactorily. Therefore, with any acceptable model which we investigated in Sect. 3, the main component in the cluster center $\left(r<0.75^{\prime}\right)$ is always a locally isothermal component and there should also exist another minor component, which may be a single phased cool component or a multiphased one. However, with the current data we cannot discriminate between these two models.

We fit the electron density profile by a double- $\beta$ model, and use the best fitting parameters to calculate the total mass. We find that within a radius of $6^{\prime}, M_{\text {tot }}=1.05 \pm 0.13 \times 10^{15} M_{\odot}$, which is consistent with the value derived by Majerowicz et al. (2002) within the error bars. We also calculate the total projected mass using a $H_{0}=100 \mathrm{~km} \mathrm{~s}^{-1} \mathrm{Mpc}^{-1}, \Omega_{\mathrm{m}}=0.3$, $\Omega_{\Lambda}=0.7$ cosmological model and compare it with what was calculated in Clowe \& Schneider (2002) by the weak lensing method. We find that the X-ray projected mass within $6^{\prime}$ is slightly lower than the weak lensing mass. However, if we assume that our best-fit $n_{\mathrm{e}}(r)$ and $T(r)$ can be extrapolated out to $15^{\prime}$ where mass measurements from Clowe \& Schneider (2002) are available, we show that the two determinations are consistent within the error bars.

Lastly, we calculate the projected mass within the optical lensing arc $(r \sim 153 \mathrm{kpc})$ using the single temperature model, which is $0.80 \pm 0.02 \times 10^{14} M_{\odot}$ and is almost the same as that from the double temperature method. This value is about half of the gravitational lensing mass. Our result is similar to that of another large cooling flow cluster PKS 0745-191 (Chen et al. 2003). Therefore, our results imply that the mass discrepancy between the X-ray and the optical lensing does exist in the well relaxed clusters. This discrepancy may be due to (i) the assumed symmetry adopted in calculating the lensing mass, and (ii) a pressure support by the radio plasma in the cluster center.

Acknowledgements. We would like to thank the referee, Stefano Ettori, for the careful reading and useful remarks. This research is partially supported by the Special Funds for Major State Basic Research Projects and the National Natural Science Foundation of China.

\section{References}

Allen, S. W. 1998, MNRAS, 296, 392

Allen, S. W. 2000, MNRAS, 315, 269

Allen, S. W., Fabian, A. C., Edge, A. C., et al. 1996, MNRAS, 283, 263

Arnaud, K. A. 1996, Astronomical data analysis software and systems V, ed. G. Jacoby, \& J. Barnes, ASP Conf. Ser., 101, 17

Arnaud, M., Neumann, D. M., Aghanim, N., et al. 2001, A\&A, 365, L80
Bartelmann, M. 1995, A\&A, 299, 11

Bevington, P. R. 1969, Data reduction and Error analysis for the Physical Sciences (New York: McGraw-Hill)

Böhringer, H., Matsushita, K., Churazov, E., Ikebe, Y., \& Chen, Y. 2002, A\&A, 382, 804

Cavaliere, A., \& Fusco-Femiano, R. 1976, A\&A, 49, 137

Chen, Y., Ikebe, Y., \& Böhringer, H. 2003, A\&A, 407, 41

Clowe, D., \& Schneider, P. 2002, A\&A, 395, 385

Dickey, J. M., \& Lockman, F. J. 1990, ARA\&A, 28, 215

Edge, A. C., Stewart, G. C., \& Fabian, A. C. 1992, MNRAS, 255, 431

Ettori, S., Fabian, A. C., Allen, S. W., \& Johnstone, R. M. 2002, MNRAS, 331, 635

Fabian, A. C. 1988, Science, 242, 1586

Fabian, A. C. 1994, ARA\&A, 32, 277

Fabian, A. C., Mushotzky, R. F., Nulsen, P. E. J., \& Peterson, J. R. 2001, MNRAS, 321, L20

Fabricant, D., Lecar, M., \& Gorenstein, P. 1980, ApJ, 241, 552

Ivison, R. J., Smail, Ian, Barger, A. J., et al. 2000, MNRAS, 315, 209

Jansen, F., Lumb, D., Altieri, B., et al. 2001, A\&A, 365, L1

Johnstone, R. M., Fabian, A. C., Edge, A. C., \& Thomas, P. A. 1992, MNRAS, 255, 431

Kaastra, J. S. 1992, An X-ray spectral code for optically thin plasma (Internal Sron-Leiden Report, updated version 2.0)

Kaastra, J. S., Lieu, R., Tamura, T., Paerels, F. B. S., \& Herder, J. W. 2003, A\&A, 397, 445

Liedahl, D. A., Osterheld, A. L., \& Goldstein, W. H. 1995, ApJ, 438, $\mathrm{L} 115$

Loeb, Ab., \& Mao, S. 1994, ApJ, 435, 109

Majerowicz, S., Neumann, D. M., \& Reiprich, T. H. 2002, A\&A, 394, 77

Makishima, K., Ezawa, H., Fukuzawa, Y., et al. 2001, PASJ, 53, 401

Markevitch, M. 2002, preprint [arXiv: astro-ph/0205333]

Matsushita, K., Belsole, E., Finoguenov, A., \& Böhringer, H. 2002, A\&A, 386, 77

Mewe, R., Gronenschild, E. H. B. M., \& van den Oord, G. H. J. 1985, A\&AS, 62, 197

Mewe, R., Lemen, J. R., \& van den Oord, G. H. J. 1986, A\&AS, 65, 511

Miralda-Escudé, J., \& Babul, A. 1995, ApJ, 449, 18

Molendi, S. 2002, ApJ, 580, 815

Molendi, S., \& Pizzolato, F. 2001, ApJ, 560, 194

Morrison, R., \& McCammon, D. 1983, ApJ, 270, 119

Nulsen, P. E. J., \& Böhringer, H. 1995, MNRAS, 274, 1093

Peres, C. B., Fabian, A. C., Edge, A. C., et al. 1998, MNRAS, 298, 416

Peterson, J. R., Paerels, F. B., Kaastra, J. S., et al. 2001, A\&A, 365, L104

Schmidt, R. W., Allen, S. W., \& Fabian, A. C. 2001, MNRAS, 327, 1057

Squires, G., Kaiser, N., Babul, A., et al. 1996, ApJ, 461, 572

Squires, G., Neumann, D. M., Kaiser, N., et al. 1997, ApJ, 482, 648

Strüder, L., Briel, U., Dennerl, K., et al. 2001, A\&A, 365, L18

Tamura, T., Kaastra, J. S., Peterson, J. R., et al. 2001, A\&A, 365, L87

Turner, M. J. L., Abbey, A., Arnaud, M., et al. 2001, A\&A, 365, L27

Voigt, L. M., Schmidt, R. W., Fabian, A. C., Allen, S. W., \& Johnstone, R. M. 2002, MNRAS, 335, 7

Weisskopf, M. C., Tananbaum, H. D., van Speybroeck, L. P., \& O’Dell, S. L. 2000, SPIE, 4012, 2

White, D. A., Jones, C., \& Forman, W. 1997, MNRAS, 292, 419 